\title{
DETERMINACIÓN DE LA CAPACIDAD DE TRANSMISIÓN POR SEMILLA DEL AMACHAMIENTO DEL FRIJOL (Aphelenchoides besseyi CHRISTIE) ${ }^{1}$
}

\author{
Néstor Felipe Chaves-Barrantes², Juan Carlos Hernández-Fonseca³, Carlos Manuel Araya-Fernández ${ }^{4}$
}

\section{RESUMEN}

Determinación de la capacidad de transmisión por semilla del amachamiento del frijol (Aphelenchoides besseyi Christie). El objetivo del presente trabajo fue determinar la capacidad de Aphelenchoides besseyi de ser transmitido por medio de la semilla en frijol. Durante el 2007 y 2008 se recolectaron semillas de plantas de frijol (cv. Cabécar) con síntomas característicos de amachamiento, en plantaciones comerciales de la región Brunca de Costa Rica (sureste del país). Entre setiembre del 2008 y setiembre del 2009, las semillas se sembraron en potes plásticos dentro de una casa de mallas en Veracruz de Pérez Zeledón, Costa Rica. Una vez emergidas, las plantas fueron observadas semanalmente para determinar la aparición de síntomas de amachamiento y detectar los casos de transmisión del nematodo en la semilla. Como respaldo a lo anterior, durante el 2014 se recolectaron semillas de plantas de frijol (cv. Cabécar) con síntomas de amachamiento, en plantaciones comerciales de cinco localidades de la región Brunca de Costa Rica. Las semillas se examinaron en un laboratorio de la Estación Experimental Agrícola Fabio Baudrit Moreno de la Universidad de Costa Rica en Alajuela, para determinar la presencia de especímenes de A. besseyi. En contraste con lo indicado para A. besseyi en arroz y pasturas, donde este patógeno se transmite por semilla, en frijol no fue posible rescatar individuos de este nematodo ni encontrar plantas enfermas a partir de semillas provenientes de plantas infestadas. Los resultados obtenidos indican que, bajo estas condiciones experimentales, la semilla de frijol no fue una vía efectiva para la transmisión y diseminación del amachamiento.

Palabras clave: falsa mancha angular, nematodo foliar, patología de semillas, Phaseolus vulgaris $\mathrm{L}$.

\begin{abstract}
Determination of the capability of common bean "amachamiento" (Aphelenchoides besseyi Christie) of being seed-transmitted. The objective of this research was to determine the capability of Aphelenchoides besseyi of being seed-transmitted in common bean. During 2007 and 2008, seeds were collected from nematode-infested common bean plants (cv. Cabécar) previously showing characteristic symptoms, in commercial plantations of the Brunca region of Costa Rica (southeastern area of the country). Between September 2008 and September 2009 the seeds were sowed in plastic pots in a greenhouse located in Veracruz, Pérez Zeledón, Costa Rica. Once seeds germinated, plants were observed weekly to determine the appearance of "amachamiento" symptoms and detect the cases of disease transmission by seed. To support the previous work, seeds from "amachamiento" diseased plants (cv. Cabécar) were collected in commercial common bean plantations in five localities of the Brunca region of Costa Rica in 2014. The seeds were carried to Estación Experimental Agrícola Fabio Baudrit Moreno of Universidad de Costa Rica located in Alajuela, where they were examined in a laboratory to determine the presence of $A$. besseyi specimens. Contrary to indicated for $A$. besseyi in rice and pastures, crops in which this pathogen is seed-transmitted, in common bean it was not possible find specimens of this nematode nor diseased plants from seeds of infested plants. The results obtained indicate that, under these experimental conditions, seeds are not an effective way for the transmission and dissemination of the "amachamiento" disease in common bean.
\end{abstract}

Keywords: false angular leaf spot, foliar nematode, Phaseolus vulgaris L., seed pathology.

1 Recibido: 22 de abril, 2015. Aceptado: 3 de junio, 2015. Proyecto de la Vicerrectoría de Investigación No. 736-A4-141 de la Universidad de Costa Rica.

2 Universidad de Costa Rica, Estación Experimental Agrícola Fabio Baudrit Moreno, Programa de Leguminosas Apdo. postal 183-4050 Alajuela, Costa Rica.nfchaves@gmail.com

3 Instituto Nacional de Innovación y Transferencia en Tecnología Agropecuaria (INTA). Apdo. 382 Centro Colón, San José, Costa Rica. jchernandez@inta.go.cr

4 Universidad Nacional, Escuela de Ciencias Agrarias, Laboratorio de Fitopatología. Apdo. postal 86-3000 Heredia, Costa Rica. carlosmanuel. araya@gmail.com 


\section{INTRODUCCIÓN}

El amachamiento del frijol es una enfermedad que ha causado reducción en el rendimiento del frijol en Costa Rica desde inicio de los años 90 (Morales et al., 1999). La enfermedad fue inicialmente diagnosticada como de naturaleza viral, causada por el virus del moteado clorótico del caupí (CCMV); sin embargo, en el 2009 se descubrió que el verdadero agente causal es el nematodo Aphelenchoides besseyi Christie (Chaves, 2011). Se caracteriza por ocasionar lesiones necróticas angulares en las hojas inferiores (Salas y Vargas, 1984; Barrantes, 2006), así como diversos grados de deformación de la nervadura central y la lámina foliar en los estratos superiores, sin mostrar algún tipo de mosaico o variegación (Araya y Hernández, 2006; Chaves, 2011; Chaves et al., 2013). Además, la planta infestada adquiere una coloración verde oscura y produce una guía anormalmente más larga (Araya y Hernández 2006), se reduce la formación de vainas entre 71 y $80 \%$ y el rendimiento entre 69 y $85 \%$ (Chaves y Araya, 2012b).

A. besseyi no sobrevive en el suelo bajo condiciones de campo (Escuer y Bello, 2000; Khan, 2004; Khan y Ghosh, 2011) y su supervivencia se da de forma anhidrobiótica en residuos de cosecha de cultivos susceptibles como frijol y arroz (Saeed y Roessner, 1984). El nematodo también se mantiene en los campos de cultivo al parasitar plantas voluntarias, malezas poáceas, y otras como Melampodium divaricatum L. Rich. ex Pers (Salas y Vargas, 1984; Prot, 1992; Escuer y Bello, 2000; Barrantes, 2006); o al darse rotaciones con otros cultivos hospedantes como maíz (Chaves y Araya, 2012a).

La semilla constituye uno de los medios más eficientes para la transmisión de organismos fitopatógenos entre períodos de siembra y a grandes distancias, incluso, entre países y continentes (Schwartz y Morales, 1994; Araya y Hernández, 2007). Esta asociación estrecha es de suma importancia epidemiológica porque favorece el establecimiento y el desarrollo de epifitias, así como la contaminación de áreas libres de un patógeno dado por medio del material de siembra (McGee, 1995; Garcia y Tenente, 2001). En el caso de $A$. besseyi, la semilla constituye la principal vía de transmisión del patógeno en pasturas como Panicum maximum (Garcia y Tenente, 2001; Bueno et al., 2002) Brachiaria brizantha (Tenente et al., 2006) y B. dictyoneura (Pinheiro et al., 1997). En arroz se presenta una situación similar, en la que el nematodo se transmite y puede sobrevivir por más de tres años de forma anhidrobiótica en las semillas (McGawley et al., 1984; Prot, 1992; Togashi y Hoshino, 2010).

A pesar de la información existente para arroz y pasturas, en frijol, cultivo en el que más del $50 \%$ de los patógenos importantes pueden ser transmitidos por semilla (Schwartz y Morales, 1994), se desconoce si $A$. besseyi se transmite por esta vía (Barrantes, 2006). Debido a la importancia epidemiológica de la potencial transmisión de este patógeno por semilla y para la implementación de prácticas de manejo de la enfermedad, se planteó el presente trabajo para determinar la capacidad de transmisión de A. besseyi por medio de la semilla en frijol.

\section{MATERIALES Y MÉTODOS}

Para estudiar la transmisión de A. besseyi en frijol, se recolectaron semillas provenientes de plantas de la variedad Cabécar con síntomas característicos de amachamiento, en plantaciones comerciales ubicadas en las comunidades de Veracruz ( $\mathrm{N} 09^{\circ} 05^{\prime}$; $\mathrm{O} 83^{\circ} 32^{\prime}$; 657 msnm) y El Águila (N 0907’; O 8331', 491 msnm), del cantón de Pérez Zeledón, provincia de San José, y Pueblo Nuevo (N 09 $07^{\prime} ;$ O 8328'; 823 msnm) y Concepción de Pilas (N 0906'; O 83²9'; 734 msnm), del cantón de Buenos Aires, provincia de Puntarenas. Cabécar es la variedad de grano rojo de mayor uso en las zonas de producción en Costa Rica. Se recolectaron 643 semillas: 40 durante el segundo ciclo de siembra del 2007 (setiembre a diciembre), 191 en el primer ciclo del año 2008 (mayo a agosto) y 412 en el segundo ciclo del 2008 (agosto a diciembre).

Entre setiembre del 2008 y setiembre del 2009, las semillas se sembraron en potes plásticos de 1000 $\mathrm{ml}$, a razón de una semilla por pote, dentro de una casa de mallas en Veracruz de Pérez Zeledón. Una vez germinadas, las plantas se examinaron semanalmente para determinar la aparición de síntomas de amachamiento, metodología similar a la utilizada para determinar la transmisión de patógenos en semillas, basada en la incidencia de plantas infectadas (Morales y Castaño, 1987; McGee, 1995).

Como respaldo a lo observado en la casa de mallas, en enero del 2015 se realizó una prueba de laboratorio en la Estación Experimental Agrícola Fabio Baudrit Moreno de la Universidad de Costa 
Rica, situada en San José de Alajuela (N 10 01'; O $84^{\circ} 16^{\prime} ; 840 \mathrm{msnm}$ ), para determinar la presencia de especímenes en semillas de frijol. Para ello, durante el 2014 se recolectaron semillas de plantas de la variedad Cabécar con síntomas característicos de la enfermedad en plantaciones comerciales, ubicadas en cinco localidades de la Región Brunca de Costa Rica (Cuadro 1), segunda en importancia para la producción de frijol del país y donde se presentan las mayores pérdidas en rendimiento por el amachamiento (Chaves y Araya, 2012b).

La presencia de $A$. besseyi se determinó mediante la adaptación de la metodología de extracción simple propuesta por Hoshino y Togashi (1999) para semillas de arroz. En cada localidad se recolectaron más de 100 semillas y se conformaron cuatro repeticiones de 25 semillas cada una. Para ello se colocaron las 25 semillas de frijol en un beaker de $150 \mathrm{ml}$, se les agregó $20 \mathrm{ml}$ de agua estéril y se dejaron reposar durante ocho horas, al cabo de las cuales se procedió a contar los especímenes de $A$. besseyi en suspensión. Para el conteo se transfirieron alícuotas de $5 \mathrm{ml}$ a un plato Petri de $50 \mathrm{~mm}$ de diámetro y con ayuda de un estereoscopio (Olympus SZ61, Japón) se determinó el número de nematodos en el agua. Los datos se analizaron con el programa Statistica 6.0 (StatSoft, Inc. 1984-2001) mediante la prueba de Kruskal-Wallis, con la que se comparó entre muestras provenientes de las distintas localidades.

\section{RESULTADOS Y DISCUSIÓN}

De las 643 semillas recolectadas de plantas con síntomas característicos de amachamiento, germinaron igual cantidad de plántulas, que se desarrollaron normalmente y sin síntomas de la enfermedad. Doce plantas $(1,86 \%)$ presentaron una deformación muy leve en las venas de los foliolos de la primera hoja trifoliada (Figura 1A), no obstante, continuaron su desarrollo de forma normal y las siguientes hojas trifoliadas brotaron sin ningún tipo de síntoma. La leve deformación que se presentó en la primera hoja trifoliada de algunas plantas pudo deberse probablemente a algún daño mecánico durante el proceso de germinación de la semilla y emergencia de la plántula, ya que no corresponde a la deformación de la vena central y de la lámina foliar características en plantas afectadas por amachamiento (Chaves, 2011; Chaves et al., 2013) (Figura 1B). Por otro lado, en ninguna de las plantas estudiadas se presentaron lesiones necróticas en las hojas cotiledonales ni en la primera trifoliada, que es uno de los síntomas iniciales del amachamiento (Chaves et al., 2013). Además, todas las plantas produjeron flores y vainas, lo que contrasta con los síntomas típicos de la enfermedad: aborto floral y producción de muy pocas vainas o su ausencia total (Chaves y Araya, 2012b; Chaves et al., 2013). La ausencia de los síntomas que pueden ser causados por A. besseyi y el desarrollo normal de todas las plantas evaluadas, indica que no hubo transmisión del nematodo a través de semilla proveniente de plantas con amachamiento.

El análisis en laboratorio de las semillas provenientes de plantas con síntomas característicos de amachamiento, corroboró lo observado en la prueba realizada en casa de mallas. Al dejar las semillas en reposo en agua estéril durante ocho horas, período al cabo del cual se extrae el $100 \%$ de los nematodos presentes en semillas de arroz, según

Cuadro 1. Localidades en donde se colectaron las semillas de plantas de frijol con síntomas característicos de amachamiento del frijol (Aphelenchoides besseyi). Región Brunca, Costa Rica. 2014.

Table 1. Localities where seeds from common bean plants showing characteristic symptoms of "amachamiento" disease were collected for the laboratory test. Brunca Region, Costa Rica. 2014.

\begin{tabular}{|c|c|c|c|c|}
\hline Localidad & Cantón & Provincia & Coordenadas & Altitud \\
\hline El Águila & Pérez Zeledón & San José & N $09^{\circ} 07^{\prime} ;$ O $83^{\circ} 32^{\prime}$ & $601 \mathrm{~m}$ \\
\hline Veracruz & Pérez Zeledón & San José & N 0906'; O 8332' & $575 \mathrm{~m}$ \\
\hline Concepción & Buenos Aires & Puntarenas & N $09^{\circ} 06^{\prime} ;$ O $83^{\circ} 29^{\prime}$ & $750 \mathrm{~m}$ \\
\hline Guagaral & Buenos Aires & Puntarenas & N 0907'; O 8350' & $444 \mathrm{~m}$ \\
\hline Chánguena & Buenos Aires & Puntarenas & N $08^{\circ} 55^{\prime} ;$ O $83^{\circ} 13^{\prime}$ & $459 \mathrm{~m}$ \\
\hline
\end{tabular}




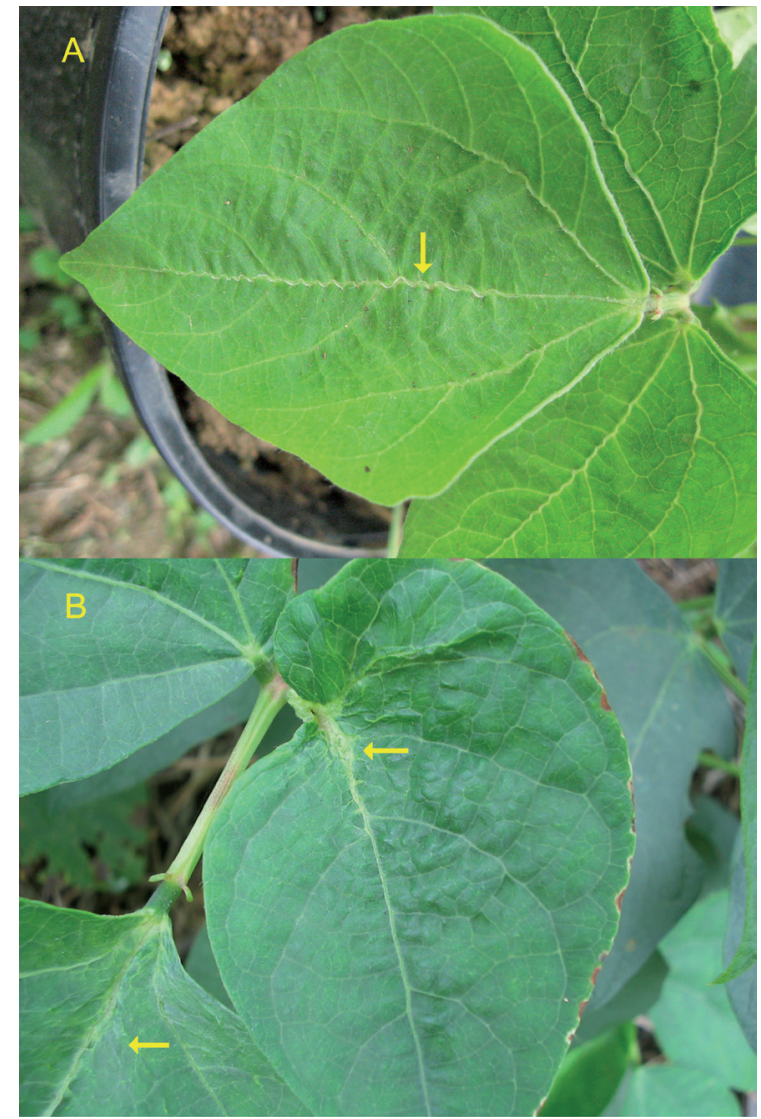

Figura 1. Síntomas observados en plantas de frijol provenientes de semillas de plantas con amachamiento causado por Aphelenchoides besseyi (A) y síntomas característicos del amachamiento del frijol (B). Veracruz, Pérez Zeledón, Costa Rica. 2008.

Figure 1. Symptoms observed in common bean plants germinated from seeds of plants with "amachamiento" disease caused by Aphelenchoides besseyi (A) and characteristic symptoms of common bean "amachamiento" (B). Veracruz, Pérez Zeledón, Costa Rica. 2008.

la metodología propuesta por Hoshino y Togashi (1999) y adaptada para frijol en este trabajo, no fue posible rescatar especímenes de A. besseyi en las 500 semillas examinadas.

La semilla de frijol se desarrolla dentro de una vaina y $A$. besseyi es un nematodo básicamente ectoparásito (Christie, 1982; Hoshino y Togashi, 1999; Proyecto Red Sicta, 2008), por lo que no tiene la capacidad de penetrar a través de las valvas y alcanzar la semilla; como sí sucede en arroz y poáceas para pastura (Prot,
1992; Pinheiro et al., 1997; Bueno et al., 2002, Tenente et al., 2006; Togashi y Hoshino, 2010). En estos cultivos, el nematodo se aloja dentro de la semilla, entre la superficie interna de la lema y la palea, y la superficie del grano, donde permanece inmóvil y latente (Christie, 1982; Escuer y Bello, 2000). Además, en vainas de frijol no se han observado síntomas debidos al ataque de A. besseyi (Proyecto Red Sicta, 2008), lo que reduce la posibilidad de que el nematodo alcance la semilla y se transmita por esta vía. Aun, en el caso hipotético de que $A$. besseyi infestara las vainas, cuando se trillan las plantas para extraer el grano, se eliminaría el material vegetal contaminado con el nematodo.

A pesar de que la semilla es el principal medio de transmisión de A. besseyi en el cultivo de arroz (McGawley et al., 1984; Saeed y Roessner, 1984; Prot, 1992; Escuer y Bello, 2000) y pasturas como Panicum maximum, Brachiaria brizantha y $B$. dictyoneura (Pinheiro et al., 1997; Garcia y Tenente, 2001; Bueno et al., 2002; Tenente et al., 2006), en frijol, bajo las condiciones experimentales descritas, no hubo transmisión del nematodo por medio de semilla. Esto porque no se consiguió rescatar especímenes de $A$. besseyi ni fue posible obtener plantas enfermas a partir de semillas de frijol provenientes de plantas con síntomas característicos de amachamiento, recolectadas en plantaciones comerciales a través de diferentes localidades y años. Los resultados obtenidos en este trabajo respaldan lo indicado por Franc et al. (1996) para $A$. ritzemabosi en frijol, que constituye una situación análoga para $A$. besseyi, en el sentido de que la única forma de que este tipo de nematodos se transmita vía semilla en frijol sería a través de la mezcla de esta con follaje enfermo o residuos de cosecha.

\section{LITERATURA CITADA}

Araya, C.M., y J.C. Hernández. 2006. Guía para la identificación de las enfermedades del frijol más comunes en Costa Rica. Ministerio de Agricultura y Ganadería (MAG), San José, CRC.

Araya, R., y J.C. Hernández. 2007. Protocolo para la producción local de semilla de frijol. Estación Experimental Fabio Baudrit Moreno, Alajuela, CRC.

Barrantes, W. 2006. Epidemiología de la falsa mancha angular (Aphelenchoides spp.) en el cultivo de frijol (Phaseolus vulgaris L.). Tesis MSc., Universidad de Costa Rica, San Pedro, CRC. 
Bueno, E.R.V., M. Prates, e R.C.V. Tenente. 2002. Avaliação de métodos tradicionais de extração de nematóides aplicados às sementes de Panicum máximum infestadas por Aphelenchoides besseyi. Nematol. Bras. 26:213-217.

Chaves, N.F. 2011. Epidemiología del amachamiento y cuantificación de las pérdidas que ocasiona en el cultivo de frijol (Phaseolus vulgaris L.). Tesis MSc., Universidad de Costa Rica, San Pedro, CRC.

Chaves, N.F., y C.M. Araya. 2012a. Efecto de la rotación de cultivos en la incidencia del amachamiento (Aphelenchoides besseyi Christie) en frijol. Agron. Costarricense 36(2):61-70.

Chaves, N.F., y C.M. Araya. 2012b. Pérdidas causadas por el amachamiento del frijol (Aphelenchoides besseyi Christie) y reacción del germoplasma comercial al patógeno. Agron. Mesoam. 23:1-12.

Chaves, N., E. Cervantes, I. Zabalgogeazcoa, y C.M. Araya. 2013. Aphelenchoides besseyi Christie (Nematoda: Aphelenchoididae), agente causal del amachamiento del frijol común. Trop. Plant Pathol. 38:243-252.

Christie, J. 1982. Nematodos de los vegetales: su ecología y control. Editorial Limusa, MEX.

Escuer, M., y A. Bello. 2000. Nematodos del género Aphelenchoides de interés fitopatológico y su distribución en España. Bol. San. Veg. Plagas 26:47-63.

Franc, G.D., C.M.S. Beaupré, F.A. Gray, and R.D. Hall. 1996. Nematode angular leaf spot of dry bean in Wyoming. Plant Dis. 80:476-477.

Garcia, J.W., e R.C.V. Tenente. 2001. Controle químico de Aphelenchoides besseyi Christie em sementes de Panicum maximum. Nematol. Bras. 25:95-98.

Hoshino, S., and K. Togashi. 1999. Simple method for determining Aphelenchoides besseyi infestation level of Oryza sativa seeds. Supplement to the J. Nematol. 31:641-643.

Khan, M.R. 2004. Observations on foliar nematode, Aphelenchoides besseyi in tuberose (Polianthes tuberosa L.). Ann. Plant Protect. Sci. 12:106-109.

Khan, M.R., and S. Ghosh. 2011. Survival and population dynamics of foliar nematode, Aphelenchoides besseyi infecting tuberose in West Bengal, India. Indian J. Nematol. 41:47-51.

McGawley, E.C., M.C. Rush, and J.P. Hollis. 1984. Ocurrence of Aphelenchoides besseyi in Louisiana rice seed and its interaction with Sclerotium oryzae in selected cultivars. J. Nematol. 16:65-68.

McGee, D.C. 1995. Epidemiological approach to disease management through seed technology. Annu. Rev. Phytopthol. 33:445-466.

Morales, F.J., and M. Castaño. 1987. Seed transmission characteristics of selected bean common mosaic virus strains in differential bean cultivars. Plant Dis. 77:51-53.

Morales, F.J., C.M. Araya, J.C. Hernández, J.A. Arroyave, M. Cuervo, A.C. Velasco, y M. Castaño. 1999. Etiología del "amachamiento" del frijol común en Costa Rica. Manejo Integrado de Plagas 52:42-48.

Pinheiro, F.P., R.P. Vianello, F.S. Ebeidalla, e R.C.V. Tenente. 1997. Uso de tratamentos térmicos na erradicação de Aphelenchoides besseyi em sementes de Brachiaria dictyoneura. Nematol. Bras. 21:92-97.

Prot, J. 1992. Diseases caused by nematodos. White tip. In: R. Webster, P. Gunnell, editors, Compendium of rice diseases. APS Press, St. Paul, MN, USA. p. 46-47.

Proyecto Red Sicta. 2008. Guía para la identificación y manejo integrado de enfermedades del frijol en América Central. Instituto Interamericano de Cooperación para la Agricultura (IICA), Managua, NCA.

Saeed, M., and J. Roessner. 1984. Anhydrobiosis in five species of plant associated nematodes. J. Nematol. 16:119-124.

Salas, L.A., y E. Vargas. 1984. El nematodo foliar Aphelenchoides besseyi Christie (Nematodo: Aphelenchoididae) como causante de la falsa mancha angular del frijol en Costa Rica. Agron. Costarricense 8(1):65-68

Schwartz, H., y F. Morales. 1994. Patología de la semilla. En: M. Pastor-Corrales, y H. Schwartz, editores, Problemas de producción del frijol en los trópicos. Segunda edición. Centro Internacional de Agricultura Tropical (CIAT), Cali, COL. p. 473-494.

Tenente, R.C.V., A.I.M. De Sousa, V.F. Gomes, and A.J.G. Rodrigues Junior. 2006. Techniques to eradicate Aphelenchoides besseyi Christie (1942) from infested Brachiaria brizantha seeds. Nematol. Bras. 30:239244.

Togashi, K., and S. Hoshino. 2010. Assesment of three-stage sampling strategy to investigate the spatial distribution and population density of Aphelechoides besseyi among Oryza sativa seeds. Nematology 12:373-380. 
